\title{
ARTICLE Profiling and targeting of cellular mitochondrial bioenergetics: inhibition of human gastric cancer cell growth by carnosine
}

\author{
Jiao-yan Cheng ${ }^{1}$, Jian-bo Yang ${ }^{1}$, Yuan Liu ${ }^{1}$, Min Xu ${ }^{1}$, Yu-yan Huang ${ }^{1}$, Jing-jing Zhang ${ }^{1}$, Pei Cao ${ }^{1}$, Jian-xin Lyu ${ }^{1,2}$ and Yao Shen ${ }^{1}$
}

\begin{abstract}
L-Carnosine ( $\beta$-alanyl-L-histidine) is a naturally occurring dipeptide distributed in various organs of mammalians. We previously showed that carnosine inhibited proliferation of human gastric cancer cells through targeting both mitochondrial bioenergetics and glycolysis pathway. But the mechanism underlying carnosine action on mitochondrial bioenergetics of tumor cells remains unclear. In the current study we investigated the effect of carnosine on the growth of human gastric cancer SGC-7901 cells in vitro and in vivo. We firstly showed that hydrolysis of carnosine was not a prerequisite for its anti-gastric cancer effect. Treatment of SGC7901 cells with carnosine $(20 \mathrm{mmol} / \mathrm{L})$ significantly decreased the activities of mitochondrial respiratory chain complexes I-IV and mitochondrial ATP production, and downregulated 13 proteins involved in mitochondrial bioenergetics. Furthermore, carnosine treatment significantly suppressed the phosphorylation of Akt, while inhibition of Akt activation with GSK690693 significantly reduced the localization of prohibitin-1 (PHB-1) in the mitochondria of SGC-7901 and BGC-823 cells. In addition, we showed that silencing of PHB-1 gene with shRNA markedly reduced the mitochondrial PHB-1 in SGC-7901 cells, and significantly decreased the colony formation capacity and growth rate of the cells. In SGC-7901 cell xenograft nude mice, administration of carnosine ( 250 mg $\mathrm{kg} / \mathrm{d}$, ip, for 3 weeks) significantly inhibited the tumor growth and decreased the expression of mitochondrial PHB-1 in tumor tissue. Taken together, these results suggest that carnosine may act on multiple mitochondrial proteins to down-regulate mitochondrial bioenergetics and then to inhibit the growth and proliferation of SGC-7901 and BGC-823 cells.
\end{abstract}

Keywords: Akt; carnosine; human gastric cancer cells; mitochondrial bioenergetics; prohibitin-1 (PHB-1)

Acta Pharmacologica Sinica (2019) 40:938-948; https://doi.org/10.1038/s41401-018-0182-8

\section{INTRODUCTION}

Gastric cancer is one of the most malignant tumors and has become the second leading cause of cancer-related death in economically developing countries. Despite the high mortality and morbidity of gastric cancer, the molecular mechanism of its initiation and progression is largely unknown. Furthermore, there is still a lack of effective therapeutic agents for the clinical treatment of gastric cancer. Therefore, discovering more effective anti-gastric cancer drugs with fewer side effects and exploring the underlying mechanisms are urgent goals.

The best studied metabolic phenotype of cancer is aerobic glycolysis, which is also known as the Warburg effect [1], and glycolysis is undoubtedly enhanced in tumors. However, a number of recent papers have shown that mitochondrial defects in tumors are rare and that adenosine triphosphate (ATP) production by glycolysis may not be necessary in every type of tumor. Mitochondrial metabolism is important for the rapid proliferation of multiple cancer cell types [2, 3], and whether the ATP for tumor cell growth is derived from aerobic glycolysis or mitochondrial bioenergetics may mainly depend on the microenvironment. Thus, targeting both mitochondrial bioenergetics and the glycolysis pathway may be a novel and effective way to inhibit the proliferation of tumor cells, including those that are resistant to conventional chemotherapeutics [4].

L-Carnosine ( $\beta$-alanyl-L-histidine), a naturally occurring dipeptide, is widely distributed in the mammalian brain, skeletal muscle, stomach, kidneys, heart, and skin [5]. Despite the fact that carnosine was characterized 100 years ago, not much is known about its physiological function. So far, several putative roles have been considered, such as its roles as an anti-oxidant, neurotransmitter, anti-inflammatory agent, mobile organic $\mathrm{pH}$ buffer, metal chelator, and wound-healing agent $[6,7]$. In recent decades, the ability of carnosine to exert inhibitory effects on the growth of tumor cells in vitro and in vivo has been demonstrated, and an inhibitory effect of carnosine on tumor cell glycolysis has been suggested [8]. Recently, our studies revealed that in addition to glycolysis, carnosine can also target mitochondrial metabolism to inhibit the proliferation of human gastric cancer cells. However, the related mechanism remains to be demonstrated [9].

Therefore, in the present study, two-dimensional electrophoresis (2-DE) followed by mass spectrometry and other common methods in pharmacology and molecular biology were used to outline the possible mechanism through which carnosine could mediate its inhibitory effects on the mitochondrial bioenergetics of human gastric cancer cells in vitro and in vivo.

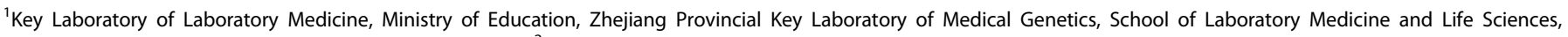
Wenzhou Medical University, Wenzhou 325035, China and ${ }^{2}$ Laboratory Medicine College, Hangzhou Medical College, Hangzhou, Zhejiang 310053, China

Correspondence: Jian-xin Lyu (jxlu313@163.com) or Yao Shen (yueshen-2002@163.com)

These authors contributed equally: Jiao-yan Cheng, Jian-bo Yang

Received: 2 April 2018 Accepted: 7 October 2018

Published online: 18 December 2018 


\section{MATERIALS AND METHODS}

Reagents

Decylubiquinone (DUB), 2,6-dichlorophenolindophenol sodium salt hydrate (DCPIP), rotenone, ubiquinone ${ }_{1}$, succinate, $\beta$-nicotinamide adenine dinucleotide, reduced dipotassium salt (NADH), malonate, carbonyl cyanide $p$-trifluoromethoxyphenyl hydrazone (FCCP), oligomycin, L-carnosine, L-histidine, $\beta$-alanine, bestatin, dithiothreitol (DTT), ethylene diamine tetracetic acid (EDTA), iodoacetate, 3-[(3-cholamidopropyl) dime thylammonio]-propanesulfonate (CHAPS), bovine serum albumin (essentially fatty acid-free), and $P^{5}$-di(adenosine) pentaphosphate were from Sigma (St. Louis, MO, USA). Penicillin, streptomycin, trypsin, L-glutamine, and trypsinEDTA solution $(0.05 \%$ (wt/vol) were bought from Invitrogen (USA). Fetal bovine serum (FBS) and Dulbecco's-modified Eagle's medium (DMEM) were from GIBCO-BRL (Grand Island, NY, USA). Lipofectamine 3000 was from Invitrogen. XF assay medium and XF calibrant solution were obtained from Seahorse Bioscience (USA). The RNA extraction kit, PrimeScript ${ }^{\mathrm{TM}}$ RT reagent kit, and SYBR Premix Ex Taq ${ }^{\mathrm{TM}}$ were bought from TakaRa Biotechnology Co., Ltd (Dalian, China). The BCA protein assay kit was bought from Beyotime Institute of Biotechnology (Nanjing, China).

\section{Animals}

All experiments using animals were performed in accordance with the National Institutes of Health Guide for the Care and Use of Laboratory Animals. Female nude mice of the age of 3-4 weeks $(10-12 \mathrm{~g})$ were used and were purchased from Shanghai SLAC Laboratory Animal Co., Ltd (Shanghai, China, Approval No. SCXK 2012-0002). The animals had access to standard chow and received water ad libitum.

\section{Cell culture and drug treatment}

The human gastric cancer cell lines SGC-7901 and BGC-823 were obtained from the Institute of Cell Biology, Chinese Academy of Sciences (Shanghai) and China Center for Type Culture Collection, CCTCC (Wuhan). The cells were cultured in DMEM supplemented with $10 \% \mathrm{FBS}, 100 \mathrm{U} / \mathrm{mL}$ penicillin $\mathrm{G}$, and $100 \mu \mathrm{g} / \mathrm{mL}$ streptomycin, and maintained at $37^{\circ} \mathrm{C}$ and $5 \% \mathrm{CO}_{2}$ in a humidified incubator. For drug treatments, $24 \mathrm{~h}$ after the cells were plated, they were treated with $20 \mathrm{mM}$ carnosine, $20 \mathrm{mM}$ histidine, $20 \mathrm{mM} \beta$-alanine, $500 \mu \mathrm{M}$ bestatin, or $20 \mathrm{mM}$ carnosine plus $500 \mu \mathrm{M}$ bestatin for $48 \mathrm{~h}$.

\section{MTT reduction assay}

Cell viability (mitochondrial activity) was monitored by the MTT reduction assay. Briefly, cells were cultured on 96-well plates with six wells in each group. At the end of the experiments, the cells were incubated with $0.5 \mathrm{mg} / \mathrm{mL} \mathrm{MTT}$ for $4 \mathrm{~h}$ at $37^{\circ} \mathrm{C}$. Then, the supernatant layer was removed, and $100 \mu \mathrm{L}$ of dimethyl sulfoxide was added into each well. MTT metabolism was quantitated spectrophotometrically at $570 \mathrm{~nm}$ in a BioRad microplate reader. The results were expressed as the percentage of MTT reduction, taking the absorbance of control cells as $100 \%$.

\section{Extracellular flux technology}

The Seahorse XF96 Flux Analyzer (Seahorse Bioscience, Billerica, MA) was also used to determine the mitochondrial metabolism activity of SGC-7901 cells under the influence of carnosine and its related agents. Here, $1.0 \times 10^{5}$ cells/well were seeded into XF96 microplates and incubated at $37^{\circ} \mathrm{C}$ for $24 \mathrm{~h}$. Then, the cells were treated with carnosine, $\beta$-alanine, L-histidine, bestatin, or carnosine plus bestatin for $48 \mathrm{~h}$. After treatment, the cells were switched to unbuffered DMEM supplemented with $2 \mathrm{mM}$ sodium pyruvate, carnosine, and its related agents $1 \mathrm{~h}$ prior to the beginning of the assay and maintained at $37^{\circ} \mathrm{C}$. After baseline measurements, oxygen consumption rates (OCRs) were measured after sequentially adding the following to each well: $20 \mu \mathrm{L}$ of oligomycin (which blocks the mitochondrial complex $\mathrm{V}$, where the electron chain is coupled to ATP synthesis), FCCP (an uncoupling agent that allows maximum electron transport), and rotenone (which blocks complex I, thereby eliminating mitochondrial respiration), to reach working concentrations of $1 \mu \mathrm{g} / \mathrm{mL}, 1 \mu \mathrm{M}$, and $1 \mu \mathrm{M}$, respectively. Each parameter, including ATP-linked OCR, proton leak, mitochondrial respiration OCR, and non-mitochondrial respiration OCR, was derived as described previously [10].

Colony formation assay

Cells were plated in six-well plates at a density of 100-200 cells per well and then were treated with carnosine or its related agents. Clones were allowed to grow for 2 weeks in DMEM supplemented with $10 \% \mathrm{FBS}, 100 \mathrm{U} / \mathrm{mL}$ penicillin $\mathrm{G}$, and $100 \mu \mathrm{g} / \mathrm{mL}$ streptomycin. Cells were subsequently fixed with $70 \%$ ethanol and stained with Coomassie Brilliant Blue for analysis of colony formation.

Purification of mitochondria and protein isolation

Mitochondria purification was conducted as described previously [11]. In short, gastric cancer SGC-7901 cells in vivo and in vitro were collected and homogenized in precooled homogenization buffer (0.25 M sucrose, $10 \mathrm{mM}$ HEPES-NaOH, pH 7.4, 1 mM EDTA). Crude mitochondria were enriched by differential centrifugation and were further purified by centrifugation in a $30-55 \%$ sucrose density gradient at $135,000 \times g$ for $15 \mathrm{~min}$. The mitochondria fraction was collected at the interface of $40 / 55 \%$ density and resuspended in mitochondria extraction buffer. An additional centrifugation at $12,000 \times g$ for 30 min was carried out to get the final purified mitochondria pellet. The mitochondria pellet was resuspended in a lysis buffer ( $30 \mathrm{mM}$ Tris-base, $7 \mathrm{M}$ urea, $2 \mathrm{M}$ thiourea, 4\% CHAPS, $65 \mathrm{mM}$ DTT, 0.2\% Bio-Lyte, $5 \mu \mathrm{L} / \mathrm{mL}$ protease inhibitor cocktail) at room temperature for $1 \mathrm{~h}$ and then centrifuged at $12,000 \times g$ at $4{ }^{\circ} \mathrm{C}$ for $30 \mathrm{~min}$. After centrifugation, the supernatant was collected for 2-DE analysis. The protein concentration was determined by the Bradford assay.

Two-dimensional electrophoresis (2-DE)

Equal amounts $(500 \mu \mathrm{g})$ of mitochondrial proteins extracted from cultured SGC-7901 cells treated with carnosine or not were pooled and diluted with rehydration buffer $(30 \mathrm{mM}$ Tris-base, $7 \mathrm{M}$ urea, $2 \mathrm{M}$ thiourea, 4\% CHAPS, $65 \mathrm{mM}$ DTT, $0.2 \%$ Bio-cye, $5 \mu \mathrm{L} / \mathrm{mL}$ protease inhibitor cocktail) for isoelectric focusing. After isoelectric focusing, the strips were first equilibrated with $130 \mathrm{mM}$ DTT in equilibration buffer $(50 \mathrm{mM}$ Tris- $\mathrm{HCl}, \mathrm{pH} 8.8,6 \mathrm{M}$ urea, $30 \%$ glycerol, $2 \%$ SDS) for $15 \mathrm{~min}$ and then with $135 \mathrm{mM}$ iodoacetamide in the same buffer for $15 \mathrm{~min}$. SDS polyacrylamide gel electrophoresis was performed with a constant current (with initial separation at a consistent $20 \mathrm{~mA} / \mathrm{gel}$ for $30 \mathrm{~min}$ followed by $50 \mathrm{~mA}$ at $20^{\circ} \mathrm{C}$ ). After $2-\mathrm{DE}$, the gels were stained with silver MS-compatible staining solution, and images were scanned for data analysis using PDQuest version 7.4.0.

In-gel digestion and mass spectrometry identification The gel pieces were destained with $50 \%$ acetonitrile (ACN)/ $25 \mathrm{mM} \mathrm{NH}_{4} \mathrm{CO}_{3}$ for $30 \mathrm{~min}$, dehydrated in $100 \%$ ACN for $10 \mathrm{~min}$, and then digested in $20 \mathrm{ng} / \mu \mathrm{L}$ trypsin solution overnight at $37^{\circ} \mathrm{C}$. After the peptide solutions were extracted with $5 \%$ TFA $/ 50 \%$ ACN, they were dried and resuspended in $5 \mu \mathrm{L}$ of $0.1 \%$ TFA for mass spectrometry analysis. Protein identification was performed on a 4700 Proteomic Analyzer MALDI-TOF-TOF mass spectrometer (Applied Biosystems) in the reflective mode. All mass spectrometry data were searched using the MASCOT search engine against a human subset of the Swiss-Prot protein sequence database.

Quantitative RT-PCR analysis

The qRT-PCR primers were as follows: PHB-1 (forward: $5^{\prime}$-gtccttg acacatctgaccttcggg-3', reverse: $5^{\prime}$-cagcagagatgatgatggccgcct- $\left.3^{\prime}\right)$; 
940

$\beta$-actin (forward: $5^{\prime}$-ccctggcacccagcac-3', reverse: $5^{\prime}$-gccgatccaca cggagtac $\left.-3^{\prime}\right)$. Total RNA was extracted from control and carnosinetreated SGC-7901 cells in vitro and in vivo and then treated with DNase I. Following reverse transcription reaction, quantitative RT-PCR was performed with Applied Biosystems StepOnePlus Real-Time PCR System using SYBR ${ }^{\circ}$ Premix Ex Taq $^{\text {TM }}$ ॥ (TaKaRa). The expression of mRNA was normalized and presented as the fold change of each mRNA in carnosine-treated samples relative to that in the controls.

\section{Western blot analysis}

Western blot analysis was carried out by a standard protocol. The following antibodies were used: rabbit anti-PHB-1 monoclonal antibody from Abcam; rabbit anti-Akt monoclonal antibody, rabbit anti-p-Akt monoclonal antibody, rabbit anti-pGSK-3 $\beta$ monoclonal antibody, rabbit anti-COX IV monoclonal antibody, and rabbit anti-histone $\mathrm{H}_{3}$ monoclonal antibody from CST Inc; and mouse anti- $\beta$-actin monoclonal antibody, mouse anti-tubulin monoclonal antibody, mouse anti-GAPDH monoclonal antibody, HRP-labeled goat anti-rabbit IgG, and HRP-labeled goat anti-mouse IgG from Beyotime Institute of Biotechnology (Nanjing, China).

Assessment of mitochondrial respiratory chain enzymatic activities Mitochondrial respiratory chain enzymatic activities (complexes I-IV) were assessed as previously described [12].

Complex I enzymatic activity. Prepared freeze-fractured mitochondria were added to the assay medium containing potassium phosphate buffer ( $\mathrm{pH} 7.5$ ), fatty acid-free $\mathrm{BSA}, \mathrm{NaN}_{3}$, and $\mathrm{NADH}$. The baseline measurement was collected at $340 \mathrm{~nm}$ for $2 \mathrm{~min}$. The reaction was started by the addition of ubiquinone $_{1}$, and the decrease in absorbance at $340 \mathrm{~nm}$ was followed using a U-3900 spectrofluorophotometer (Hitachi) for $2 \mathrm{~min}$. In parallel, separate cuvettes containing the same quantity of reagents and sample but not rotenone were also analyzed. In this assay, the rotenone-sensitive activity was considered to be specific complex I activity.

Complex II enzymatic activity. Prepared mitochondria were added to the assay medium containing potassium phosphate buffer, fatty acid-free BSA, $\mathrm{NaN}_{3}$, succinate, and DCPIP. The mixture was incubated inside the spectrophotometer at $37^{\circ} \mathrm{C}$ for $8 \mathrm{~min}$, and then baseline activity was read at $600 \mathrm{~nm}$ for $2 \mathrm{~min}$. The reaction was begun by the addition of DUB, and the decrease in the absorbance at $600 \mathrm{~nm}$ was recorded for $2 \mathrm{~min}$. In parallel, malonate was added to cuvettes. The complex II-specific rate was determined by subtracting the malonate-insensitive activity from the total activity.

Complex III enzymatic activity. Prepared mitochondria were added to the assay medium containing potassium phosphate buffer, oxidized cytochrome $c, \mathrm{NaN}_{3}$, EDTA, and Tween-20. Baseline at $550 \mathrm{~nm}$ was read for 2 min before the addition of decylubiquinol. The samples were mixed rapidly, and then the increase in absorbance at $550 \mathrm{~nm}$ was immediately observed for $2 \mathrm{~min}$. In parallel, separate cuvettes containing the same quantity of reagents and samples with the addition of antimycin A were also prepared. The antimycin A-sensitive activity was considered to be the specific complex III activity.

Complex IV enzymatic activity. Reduced cytochrome $c$ was added to the potassium phosphate buffer, and baseline activity at $550 \mathrm{~nm}$ was read for the last $2 \mathrm{~min}$. The reaction was begun by the addition of prepared mitochondria, and the decrease in absorbance at $550 \mathrm{~nm}$ was read for $2 \mathrm{~min}$. The specificity of complex IV activity was checked by a parallel experiment in which $\mathrm{NaN}_{3}$ was added.
Measurement of mitochondrial ATP synthesis

Mitochondrial ATP was measured as previously reported [13]. In brief, after treatment, mitochondria were isolated from SGC-7901 cells and resuspended in buffer A (containing $150 \mathrm{mM} \mathrm{KCl}, 25 \mathrm{mM}$ Tris-HCl, $2 \mathrm{mM}$ EDTA, $0.1 \%$ bovine serum albumin, $10 \mathrm{mM}$ potassium phosphate, $0.1 \mathrm{mM} \mathrm{MgCl}_{2}, \mathrm{pH} 7.4$ ) at room temperature. After $50 \mu \mathrm{g}$ pelleted mitochondria were resuspended in $160 \mu \mathrm{L}$ of buffer $\mathrm{A}$ and $0.15 \mathrm{mM} P^{1}, P^{5}$-di(adenosine) pentaphosphate, either $1 \mathrm{mM}$ malate plus $1 \mathrm{mM}$ pyruvate or $5 \mathrm{mM}$ succinate plus $2 \mu \mathrm{g} / \mathrm{mL}$ rotenone, $10 \mu \mathrm{L}$ of buffer B (containing $0.8 \mathrm{mM}$ luciferin and $20 \mathrm{mg} / \mathrm{mL}$ luciferase in $0.5 \mathrm{M}$ Tris-acetate $\mathrm{pH} 7.75$ ), and $0.1 \mathrm{mM}$ ADP were added. For each sample, one replicate tube was prepared containing the above components plus $10 \mu \mathrm{g} / \mathrm{mL}$ oligomycin. Luminance was measured by a monochromator microplate reader. Standard curves were generated, and the protein concentration of each treatment group was determined using the BCA protein assay kit. ATP synthesis was expressed as nanomoles of ATP synthesized per minute per milligram of mitochondrial protein.

\section{Gene silencing}

An shRNA plasmid targeting PHB (Forward: 5'-GATCCCCCGTGGG TAC AGAAACCA ATTCTCGA GAATTGGTTTCTGTACCCACGGTITITG GAT-3'; Reverse: 5'-AGCTATCC AAAAACCGTGGGTACAGAAACCA ATTCTCGAGAATTGGTITCTGT ACCCACGGG G-3') was used in this study. SGC-7901 cells were transfected with shRNA or scrambled negative control shRNA using Lipofectamine 3000 according to the transfection protocol provided by the manufacturer (Invitrogen). The mRNA and protein expression levels of PHB-1 were measured using $\mathrm{QPCR}$ and western blot (see above).

\section{Statistical analysis}

All data represent three or more independent experiments. Data were expressed as the mean $\pm S D$. Statistical analyses were conducted by SPSS 11.5 for Windows. One-way ANOVA (analysis of variance) followed by LSD (least significant difference) or Dunnett's T3 post hoc test (where equal variances were not assumed) was applied for multiple comparisons, whereas Student's $t$-test was used for comparisons between two groups. $P<0.05$ was considered statistically significant.

\section{RESULTS}

Effects of carnosine and its related agents on mitochondrial bioenergetics and colony formation of cultured SGC-7901 and BGC-823 cells

An aminopeptidase inhibitor bestatin, $\beta$-alanine, and L-histidine were used to determine whether the inhibitory effect of carnosine on mitochondrial bioenergetics and the proliferative capacity of human gastric cancer cells is mediated by one of its components, $\beta$-alanine or L-histidine, through hydrolysis. As seen in Fig. 1a-c, treatment with $20 \mathrm{mM}$ carnosine for $48 \mathrm{~h}$ markedly reduced the basal OCRs, ATP-linked OCRs, and mitochondrial respiration OCRs as measured by the Seahorse XF96 Extracellular Flux Analyzer. Histidine but not $\beta$-alanine mimicked the effect of carnosine on the mitochondrial respiration of SGC-7901 cells, significantly reducing the basal OCRs and the ATP-linked and mitochondrial respiration OCRs by $22.9 \%, 31.1 \%$, and $27.34 \%$, respectively. Bestatin alone did not affect the mitochondrial respiration of SGC-7901 cells, and when it was applied together with carnosine, bestatin was unable to reverse the inhibitory effect of carnosine on the mitochondrial respiration of SGC-7901 cells.

In the colony formation assay, chronic exposure to carnosine reduced colony formation to $14.5 \%$ and $18.6 \%$ of control in cultured SGC-7901 and BGC-823 cells, respectively. $\beta$-Alanine did not affect colony formation of cultured SGC-7901 cells, whereas histidine treatment significantly inhibited colony formation as almost no colony was detected in the histidine-treated group. 
a

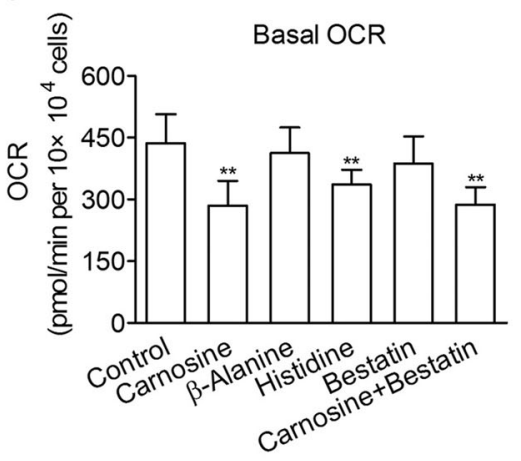

C



e

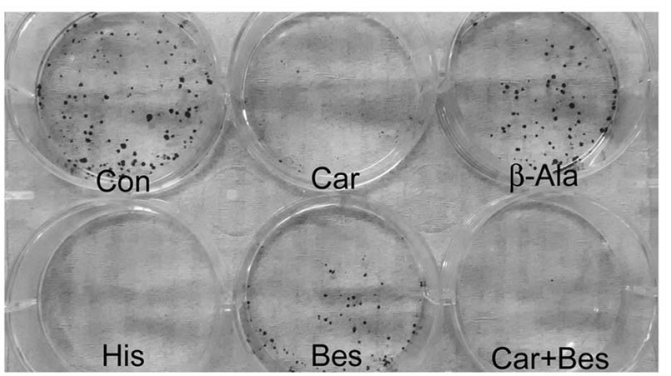

g

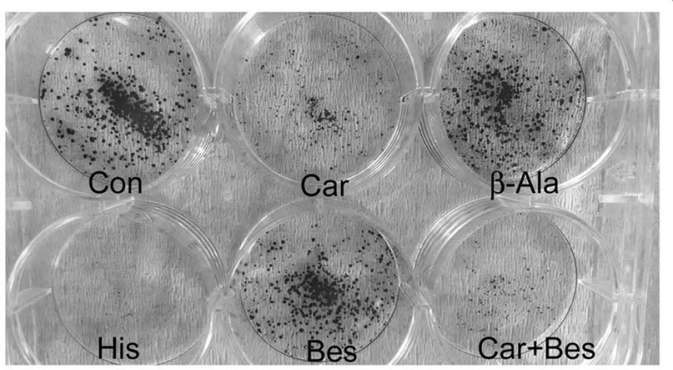

b

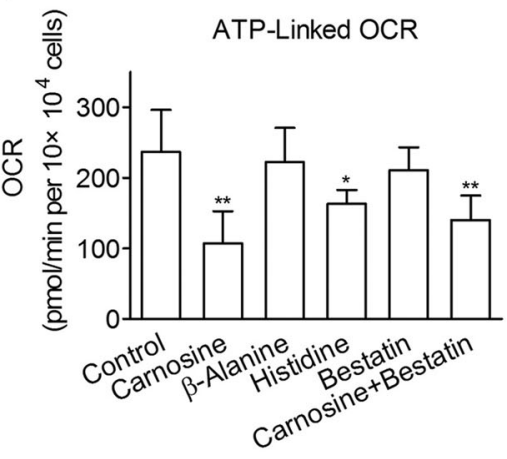

d
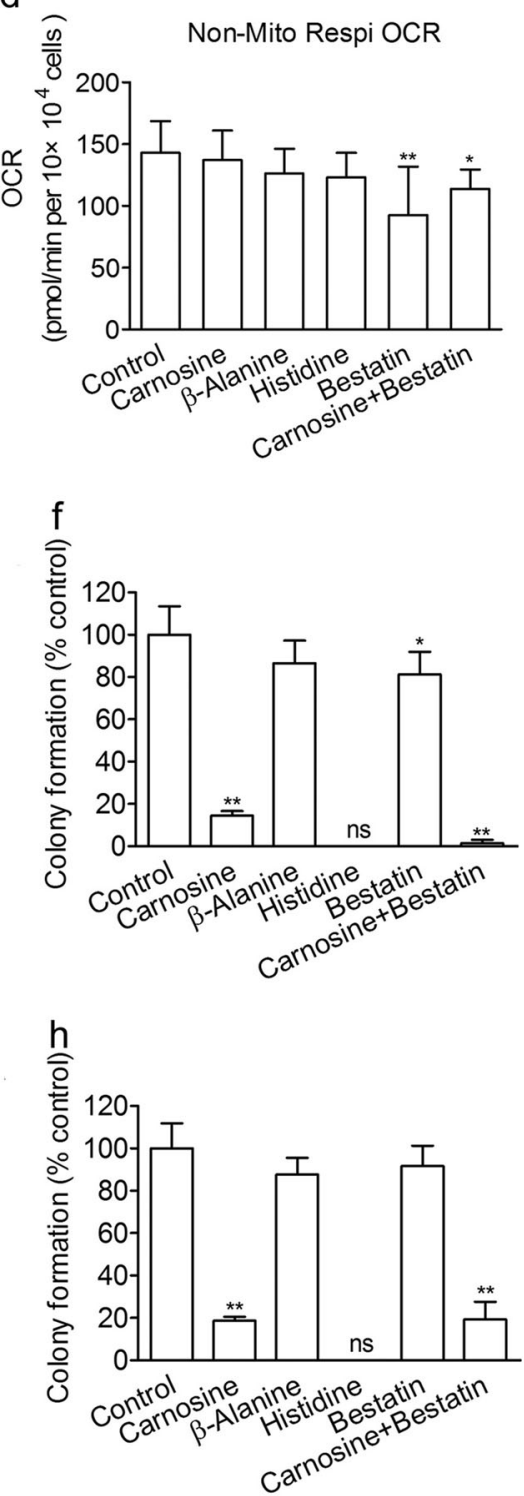

Fig. 1 Effects of carnosine and its related agents on mitochondrial respiration and the proliferative capacity of cultured SGC-7901 cells. Realtime analysis of oxygen consumption rates (OCRs) of cultured SGC-7901 cells after perturbation with small-molecule metabolic modulators under the influence of carnosine, $\beta$-alanine, histidine, bestatin, and carnosine plus bestatin. Oligomycin $(\mathrm{O}, 1 \mu \mathrm{M})$, FCCP $(\mathrm{F}, 1 \mu \mathrm{M})$, and rotenone $(R, 1 \mu \mathrm{M})$ were injected sequentially into each well after measurement of the baseline rate. a Basal OCR, b ATP-linked OCR, $\mathbf{c}$ mitochondrial respiration OCR, and $\mathbf{d}$ non-mitochondrial respiration OCR are shown. Representative images of the cloning wells of SGC-7901 cells (e) and BGC-823 cells (g). Cells were seeded at a low density in DMEM supplemented with carnosine, $\beta$-alanine, histidine, bestatin, and carnosine plus bestatin for 14 days. The colonies were subsequently fixed with $70 \%$ ethanol and stained with Coomassie Brilliant Blue for analysis of colony formation. Quantitative image analysis of colonies in cultured SGC-7901 cells (f) and BGC-823 cells (h). Data are expressed as the mean \pm SD. $n=6-10 .{ }^{*} P<0.05,{ }^{* *} P<0.01$ vs. the control group 
Bestatin alone reduced colony formation to $81 \%$ and $91.6 \%$ of control in SGC-7901 and BGC-823 cells, respectively, and its combination with carnosine inhibited colony formation to $1.4 \%$ and $19.2 \%$ of control in SGC-7901 and BGC-823 cells, respectively (Fig. 1e-h).

Carnosine inhibited gastric tumor growth in vivo

In the present work, we also investigated whether carnosine also affects gastric tumor growth in vivo. In two independent experiments, $2 \times 10^{6}$ SGC-7901 cells in $200 \mu \mathrm{L}$ sterile phosphatebuffered saline were subcutaneously injected into the right inguinal fat pad of female nude mice. After 1 week, once a subcutaneous tumor had formed, the animals in each of the two series were randomly divided into four groups. Groups 1-3 received a daily intraperitoneal injection of $500 \mu \mathrm{L}$ carnosine $(5,50,250 \mathrm{mg} / \mathrm{kg}$ body weight respectively) dissolved in $\mathrm{NaCl}$ solution $(0.7 \%), \mathrm{pH} 7.4$. Group 4 was used as a control and received a daily injection of $500 \mu \mathrm{L} 0.9 \% \mathrm{NaCl}, \mathrm{pH}$ 7.4. All injections were performed for 3 weeks. Tumor weight was assayed at the end of the experiment, and tumor size was determined every 2 days after injection. Tumor size was determined by measuring the maximum and minimum diameters with a caliper rule, and the product of these two diameters was defined as the tumor size with the units $\mathrm{mm}^{2}$ [14]. The results of the experiments are presented in Fig. 2. As seen, carnosine at low concentrations of 5 and $50 \mathrm{mg} / \mathrm{kg}$ body weight did not influence the development of the subcutaneous tumor. However, carnosine at a high concentration of $250 \mathrm{mg} / \mathrm{kg}$ body weight significantly inhibited subcutaneous tumor development as the tumor weight was significantly lower at the end of the experiments in the group treated with $250 \mathrm{mg} / \mathrm{kg}$ carnosine than in the control group, and the tumor volume was obvious smaller in the $250 \mathrm{mg} / \mathrm{kg}$ carnosine-treated group than in the control group beginning on day 12 after the start of the carnosine injections.

Carnosine inhibited mitochondrial respiratory chain activity and mitochondrial ATP synthesis in cultured SGC-7901 cells

In the current study, we also detected the activity of the mitochondrial respiratory chain complexes I-IV in SGC-7901 cells under the influence of carnosine. The results showed that carnosine treatment markedly reduced the activities of mitochondrial respiratory chain complexes I-IV (complex I, control group: $972.38 \pm 170.41$; carnosine-treated group: $542.23 \pm 46.75 ; P<0.05$; complex II, control group: $644.4 \pm 48.09$; carnosine-treated group: $401.02 \pm 18.37$; $P<0.01$; complex III, control group: $559.81 \pm 121.29$; carnosine-treated group: $262.56 \pm 63.55, P<0.01$; complex IV, control group: $1891.97 \pm 109.84$; carnosine-treated group: $1481.76 \pm 16.63$, $P<0.01$; Fig. 3a). We further determined the effect of carnosine on the production of mitochondrial ATP and found that with both malate/pyruvate and succinate as substrates, ATP synthesis was significantly lower in carnosine-treated SGC-7901 cells than in the respective control groups (malate/pyruvate control group: $308.0 \pm$ 37.3 ; malate/pyruvate carnosine-treated group: $247.2 \pm 32.7$; $P<0.05$; succinate control group: $225.7 \pm 22.7$; succinate carnosinetreated group: $200.3 \pm 14.1, P<0.05$; Fig. $3 b$ ).

Comparative mitochondrial proteomic profiling between carnosine-treated and untreated SGC-7901 cells

As seen in Fig. 4, representative gel images were selected for comparative mitochondrial proteomic analyses of SGC-7901 cells treated with and without carnosine $(20 \mathrm{mM})$ for $48 \mathrm{~h}$. A total of 363 differentially expressed proteins were found. Among them, 13 proteins were expressed only in the controls, and 283 proteins were downregulated and 67 were upregulated under the influence of carnosine (data not shown). Spots with two-fold variation between the two groups were selected to be analyzed by mass spectrometry for further study. A total of 13 differentially expressed proteins involved in mitochondrial metabolism

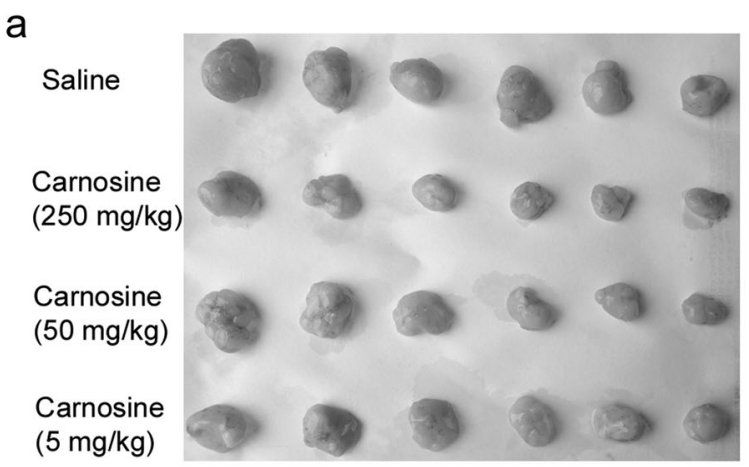

b

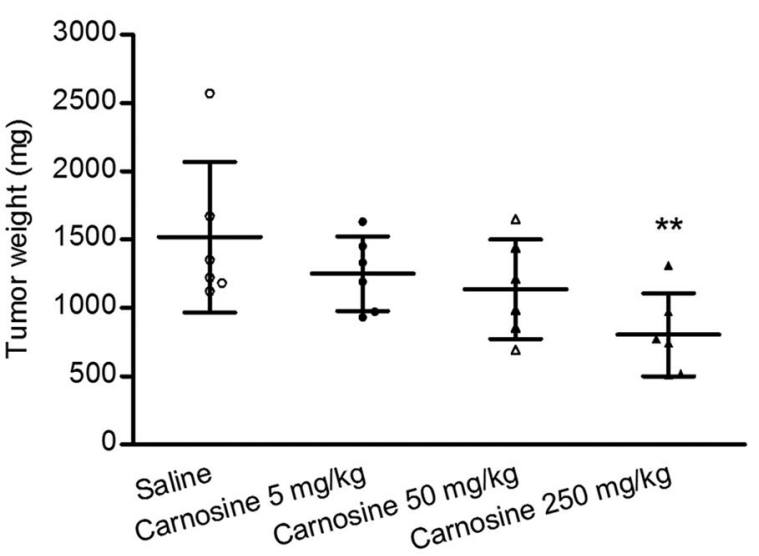

C



Fig. 2 Carnosine inhibited the development of SGC-7901 tumors in vivo. a The images show the tumor tissues from the animals at day 20 after carnosine treatment. $\mathbf{b}$ The tumor weight was measured at the end of the experiment. c The size of tumors developed from subcutaneously implanted SGC-7901 cells was determined by measuring the maximum and minimum diameters. The tumor size presented is the product of these two diameters measured at different days after the start of the carnosine injection. Data are expressed as the mean \pm SD. $n=6 .{ }^{*} P<0.05,{ }^{* *} P<0.01$ vs. the saline group

pathways were successfully identified, and all 13 of the proteins were downregulated in the carnosine-treated samples. Details of the identification of the 13 differently expressed proteins, accession numbers, experimental PI value, molecular weight, number of matched peptides, sequence coverage, protein score, and functional classification are shown in Table 1. 


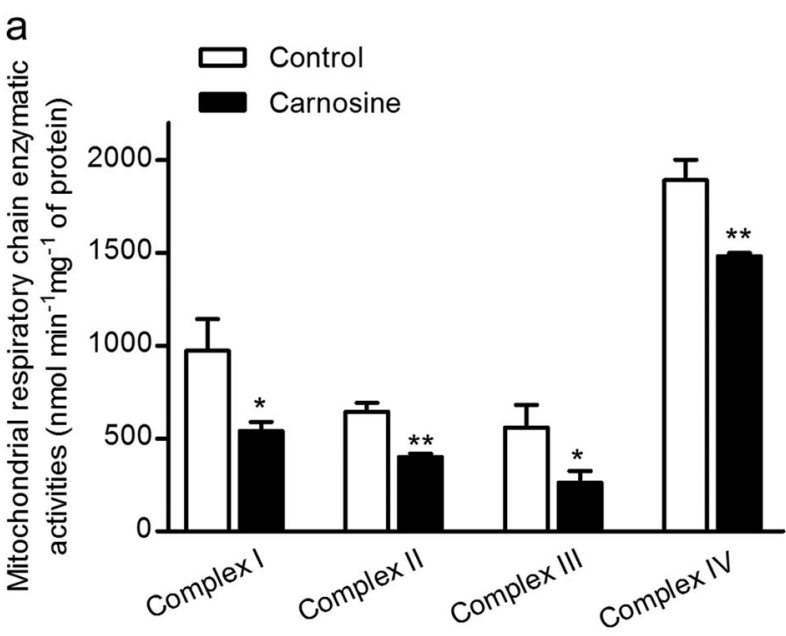

b

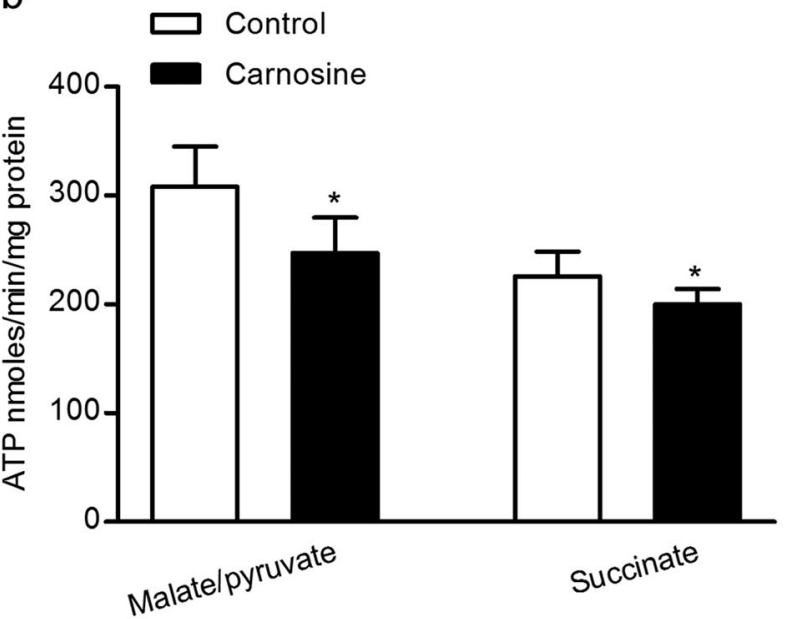

Fig. 3 Carnosine inhibited the activity of the mitochondrial respiratory chain and mitochondrial ATP production in cultured SGC-7901 cells. The cells were treated with carnosine $(20 \mathrm{mM})$ for 48 $\mathrm{h}$ and then were harvested to isolate mitochondria to measure the activities of the mitochondrial respiratory chain complexes I-IV (a) and mitochondrial ATP production (b). Data are expressed as the mean \pm SD. $n=3-6$. ${ }^{*} P<0.05,{ }^{* *} P<0.01$ vs. the control group

Effects of carnosine on the expression of mitochondrial PHB-1 in vitro and in vivo

Mitochondrial PHB-1 plays an important role in maintaining normal mitochondrial function [15, 16], and loss of mitochondrial PHB-1 has been suggested to be able to lead to impairment of the mitochondrial respiratory chain. Therefore, the differentially expressed protein PHB-1 was selected to be validated in both in vitro and in vivo samples by immunofluorescence and western blot. The immunofluorescence results showed that PHB-1 was predominantly localized in the mitochondria with only a small portion of PHB-1 localized in the nucleus in cultured SGC-7901 cells. Carnosine treatment markedly reduced the mitochondrial localization of PHB-1. The western blot results were consistent with the immunofluorescence data as shown in Fig. 5.

Carnosine reduced the mitochondrial localization of PHB-1 through repressing Akt activation

The Akt signaling pathway is one of the most frequently dysregulated pathways in cancer and is thought to have a major role in human gastric cancer [17]. Interestingly, previous studies have shown that phosphorylation of PHB-1 directly by Akt mediates the mitochondrial localization of PHB-1 and promotes the proliferation of bladder cancer cells [18], but its relationship with human gastric cancer remains unknown. To examine whether the activity of Akt is responsible for localization of PHB-1 in human gastric cancer, SGC-7901 and BGC-823 cells were treated with the Akt inhibitor GSK690693. As shown in Fig. 6a, a decrease in phosphorylation of the Akt substrate GSK3 (Ser9) and an increase in Akt phosphorylation at the Ser473 site were observed in SGC7901 and BGC-823 cells treated with GSK690693, consistent with the feedback mechanism observed previously with this Akt kinase inhibitor. Furthermore, inhibition of Akt by GSK690693 effectively reduced the localization of PHB-1 in mitochondria. In the current study, we found that carnosine treatment induced a marked decrease in the phosphorylation status of Akt in SGC-7901 and BGC-823 cells (Fig. 6b). In addition, carnosine treatment decreased the localization of PHB-1 in mitochondria (Fig. 6b). Taken together, these findings suggest that carnosine may reduce the localization of PHB-1 in mitochondria by inhibiting Akt pathway activation.

Mitochondrial PHB-1 regulated the proliferative capacity of SGC7901 cells

To further explore the involvement of mitochondrial PHB-1 in the regulation of human gastric cancer cell growth, PHB shRNA was transfected in SGC-7901 cells. qPCR and western blot results showed that the gene silencing was successful, and PHB-1 was significantly reduced in mitochondrial fractions in stable transfectants (Fig. 7a-d). Then, the growth rate and colony formation capacity of the cells were assayed. As shown in Fig. 7e-g, loss of PHB-1 (mainly mitochondrial PHB-1) significantly inhibited the growth rate and colony formation capacity of SGC-7901 cells, while untreated shPHB transfectants exhibited a cell growth rate and colony formation capacity comparable to control cultures.

\section{DISCUSSION}

In this study, we demonstrated an inhibitory effect of carnosine and its underlying mechanism on mitochondrial bioenergetics of human gastric cancer cells in vitro and in vivo. Our principal findings are as follows. First, carnosine inhibited SGC-7901 cell growth both in vivo and in vitro. Second, hydrolysis of carnosine was not a prerequisite for its anti-human gastric cancer effect. Third, multiple mitochondrial proteins related with mitochondrial bioenergetics may be involved in the inhibitory action of carnosine in human gastric cancer cells. Fourth, carnosine may inhibit the phosphorylation of Akt to reduce the mitochondrial localization of PHB-1, which plays an important role in the regulation of human gastric cancer cell growth. Therefore, carnosine should be considered along with the growing armament of compounds in various stages of drug development that target tumor metabolism, one of the key hallmarks of tumors.

Currently, the mode of carnosine action has not yet been elucidated, and whether the inhibitory effect of carnosine is mediated by one of its components, L-histidine or $\beta$-alanine, and whether the hydrolysis of the dipeptide is a prerequisite for its anti-neoplastic effect are not known. In the current study, we detected the mitochondrial activity and the proliferative capacity of SGC-7901 and BGC-823 cells treated with carnosine, bestatin, $\beta$-alanine, and L-histidine. Bestatin has been used as an adjuvant chemotherapy drug [19]. In addition, bestatin inhibits carnosinase, which is expressed in tumor cells and hydrolyzes carnosine into its components $\beta$-alanine and L-histidine $[20,21]$. We found that treatment with bestatin alone for 2 days did not affect mitochondrial activity but showed a mild inhibitory effect on colony formation after a chronic exposure for 14 days in cultured SGC-7901 cells. The antiproliferative effect of carnosine was not reversed but was rather enhanced when it was used in combination with bestatin. Thus, the data suggest that the inhibitory effects of carnosine on the mitochondrial bioenergetics and proliferative capacity of cultured SGC-7901 cells was due to 

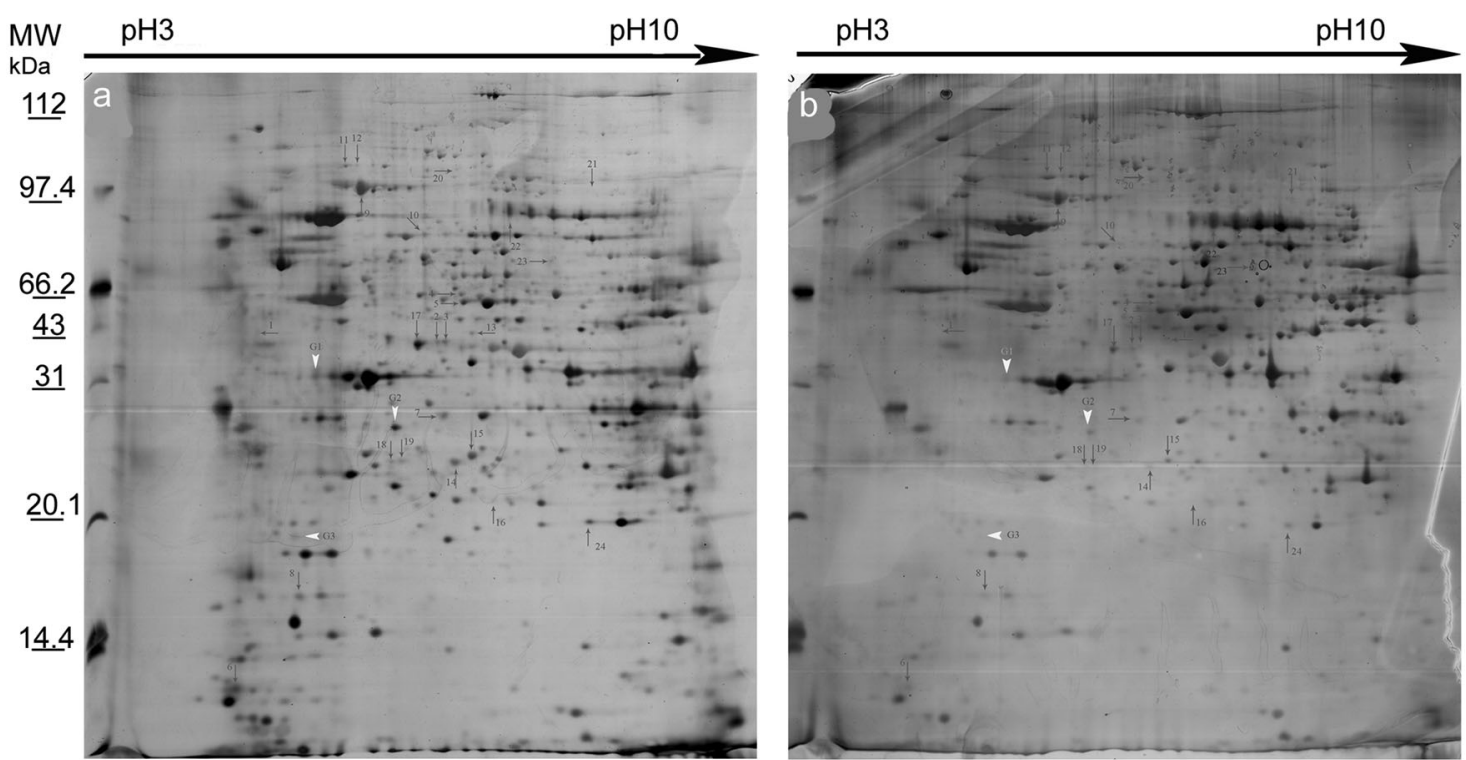

Fig. 4 Representative images of two-dimensional electrophoresis (2-DE). 2-DE maps of mitochondrial proteins of cultured SGC-7901 cells treated with and without carnosine $(20 \mathrm{mM})$ for $48 \mathrm{~h}$. a Control cells and (b) carnosine-treated cells. Numbers indicated on the map highlight several of the protein spots that were differentially expressed

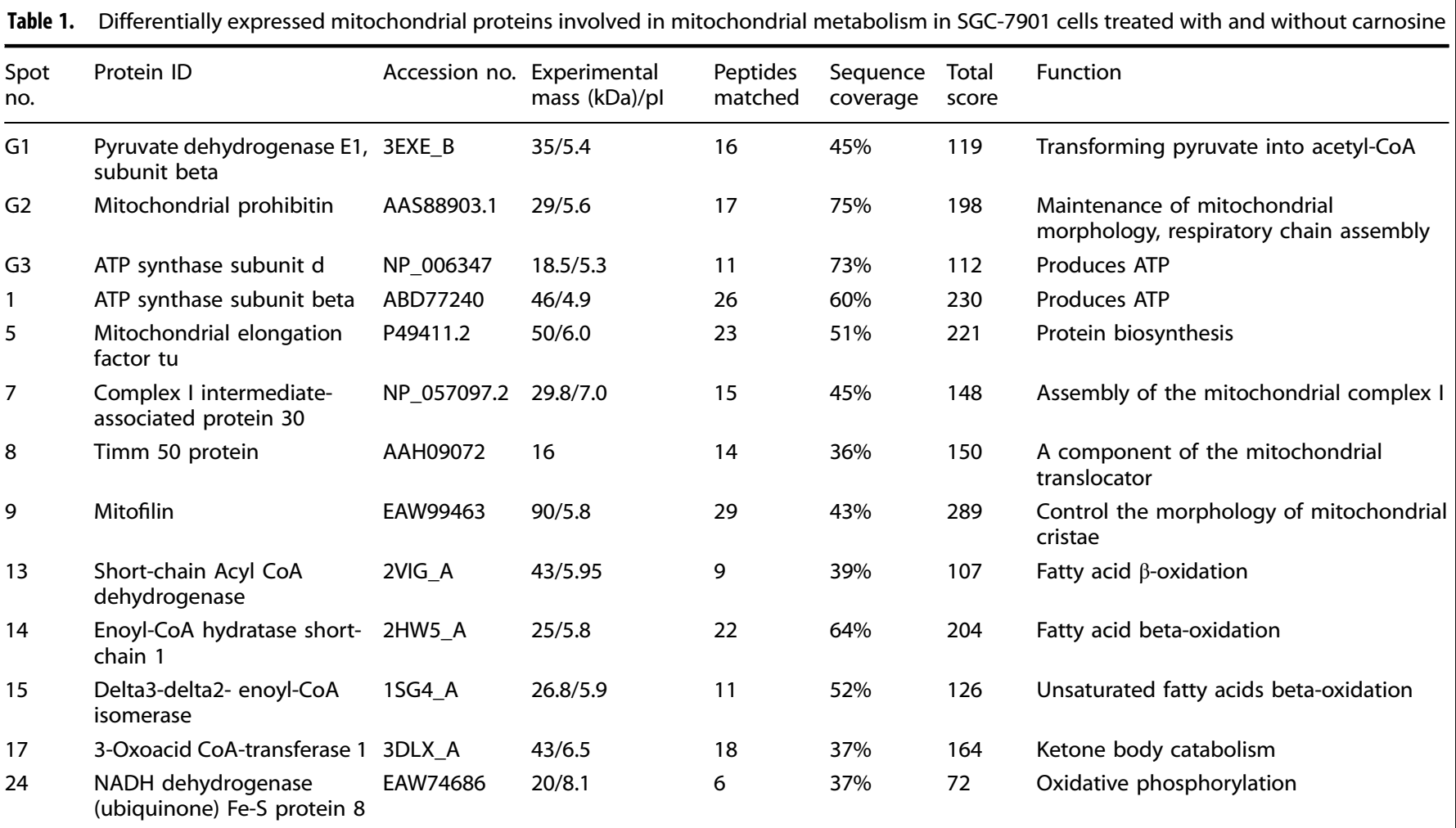

its own action but not to its components through hydrolysis, although histidine also exerted a potent inhibitory effect on mitochondrial activity and proliferative capacity in cultured SGC-7901 and BGC-823 cells.

Carnosine has been shown to have a high potential to inhibit growth of malignant cells in vivo when given at the time of subcutaneous cancer cell injection into the dorsal skin of female nude mice [14]. In the present study, we found that carnosine at a high concentration markedly inhibited SGC-7901 cell growth in vivo even when given at 1 week after the cancer cells were injected into the nude mice when the subcutaneous tumor had already formed. We speculated that this effect was probably due to the presence of serum carnosinase, which is considered to be an impediment to the therapeutic use of carnosine [22]. To date, a number of strategies that could overcome this perceived obstacle have been suggested, including the use of forms of carnosine resistant to carnosinase attack and the use of an intranasal delivery route to combat neurodegeneration and brain tumors [22]. In addition, carnosine is a naturally occurring dipeptide and possess anti-oxidant and anti-inflammatory 
a

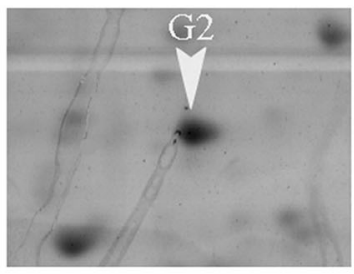

Control

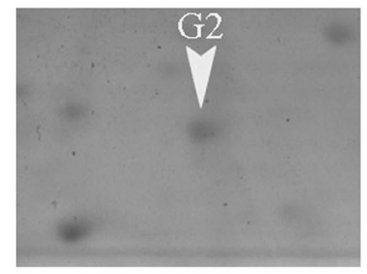

Carnosine b
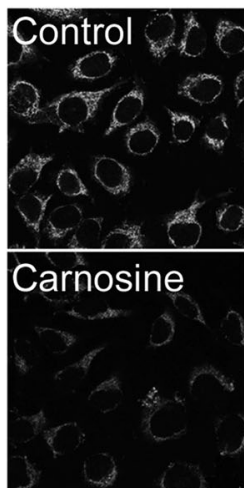
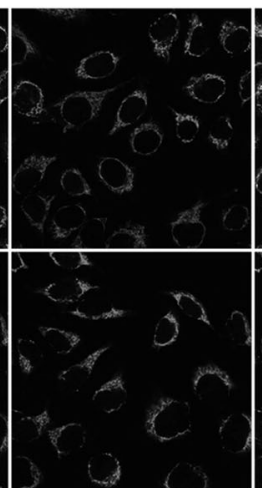

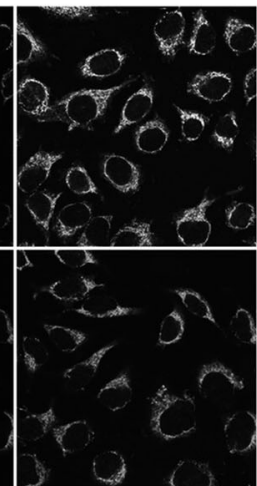

C
In vitro

Mito-PHB-1

\section{COXIV}
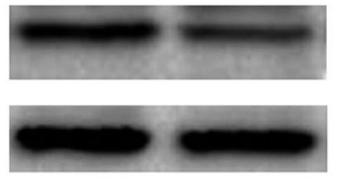

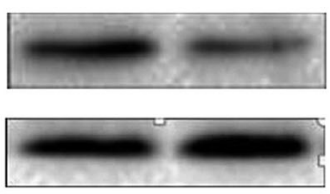

In vivo d

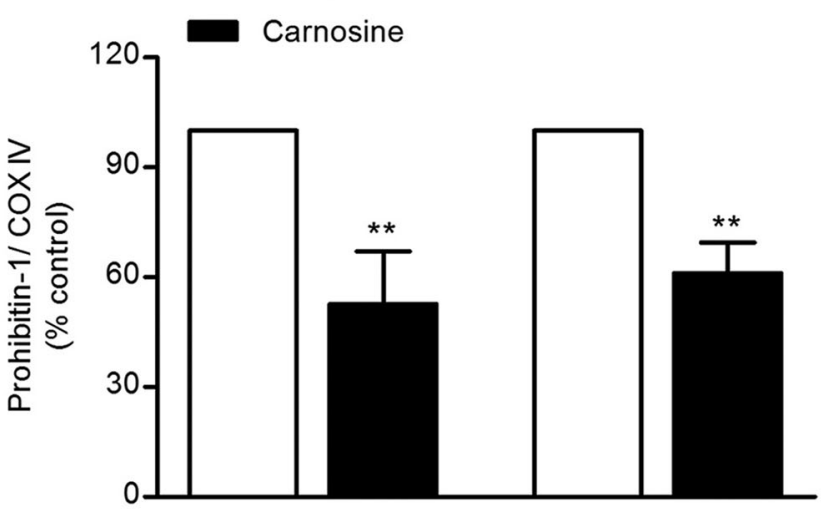

In vitro

Fig. 5 Effects of carnosine on the expression of mitochondrial PHB-1 in vitro and in vivo. a Detailed alteration pattern of mitochondrial PHB-1 in control and carnosine-treated SGC-7901 cells from two-dimensional electrophoresis. b Immunofluorescence analysis of mitochondrial PHB1 expression in cultured SGC-7901 cells exposed to carnosine $(20 \mathrm{mM})$ for 48 h. Green: PHB-1; red: Mito Tracker ${ }^{\circledR}$ Red CMXRos; confocal images were obtained ( $\times 600$ objective). c Western blot analysis of mitochondrial PHB-1 protein levels in cultured SGC-7901 cells treated with carnosine for $48 \mathrm{~h}$ (in vitro) and in tumor tissues from the animals at day 20 after carnosine treatment (in vivo). $\mathbf{d}$ Densitometric analysis of bands showing relative mitochondrial PHB-1 expression levels in vitro and in vivo. Data are expressed as the mean \pm SD. $n=5-6$. ${ }^{* *} P<0.01$ vs. the saline group

a

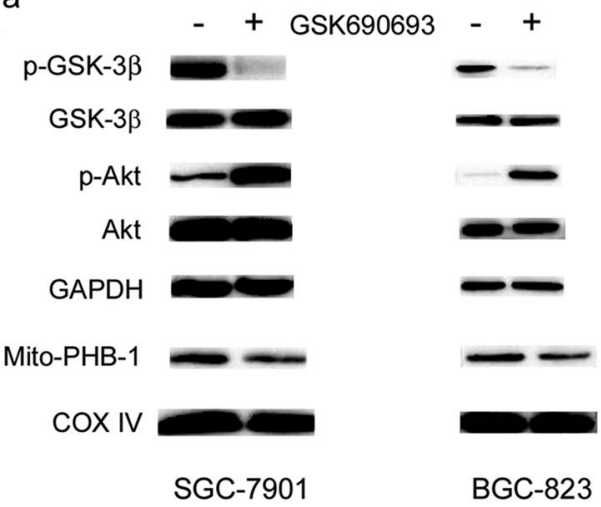

b

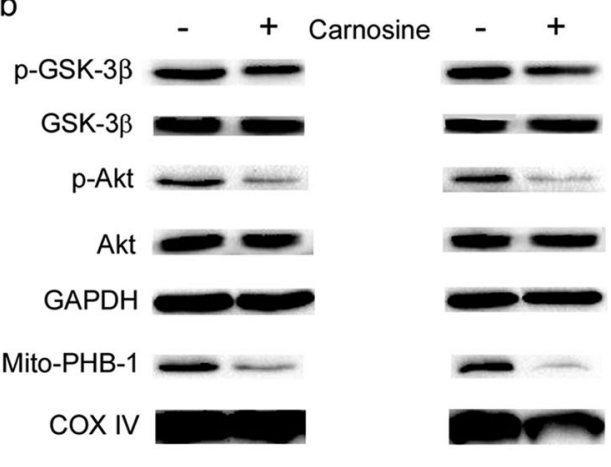

SGC-7901

BGC-823

Fig. 6 Effects of GSK690693 and carnosine on the expression of mitochondrial PHB-1 in cultured SGC-7901 and BGC-823 cells. Western blot analysis of the expression levels of Akt signaling pathway proteins and mitochondrial PHB-1 in cultured SGC-7901 and BGC-823 cells with or without GSK690693 (a) and carnosine (b)

activities, even if carnosine at a high concentration has little side effects. Thus, carnosine may be a potential agent with minor side effects for human gastric cancer therapy.

Cancer cells adapt to various microenvironments and energyrelated challenges by switching between glycolysis and mitochondrial metabolism. Metabolic reprogramming provides the cellular energy required for increased cancer growth. Recently, targeting the glycolysis pathway and the mitochondrial bioenergetics in cancer cells has shown to be an attractive and effective chemotherapeutic strategy, even in slow-growing hypoxic tumor cells normally resistant to conventional chemotherapeutic agents [23]. Early on, carnosine was reported to inhibit growth of transformed cells through, at least in part, depleting glycolytic ATP levels [22, 24]. Recently, our study found that in addition to targeting the glycolysis pathway, carnosine also inhibits the mitochondrial bioenergetics of cultured human gastric cancer SGC-7901 cells [9]. However, the molecular targets have not yet been elucidated. Comparative proteomic strategies provide useful tools in identifying novel molecular targets for anticancer therapy. In the current study, a panel of proteins involved in mitochondrial 
a

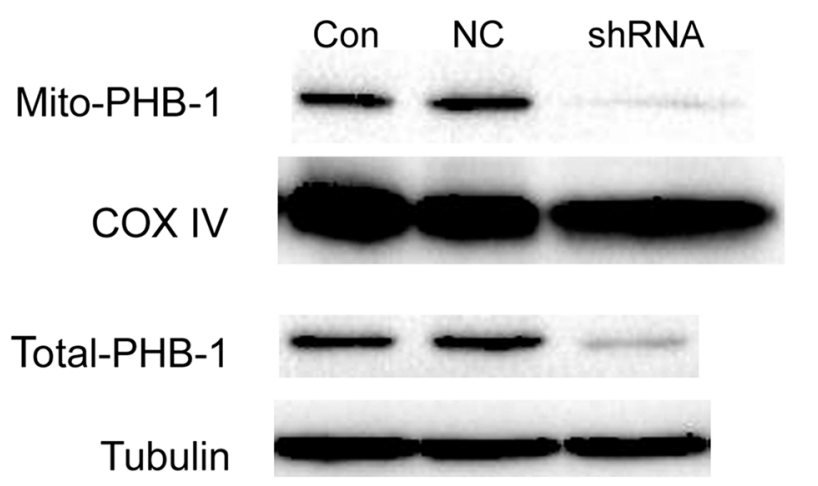

C

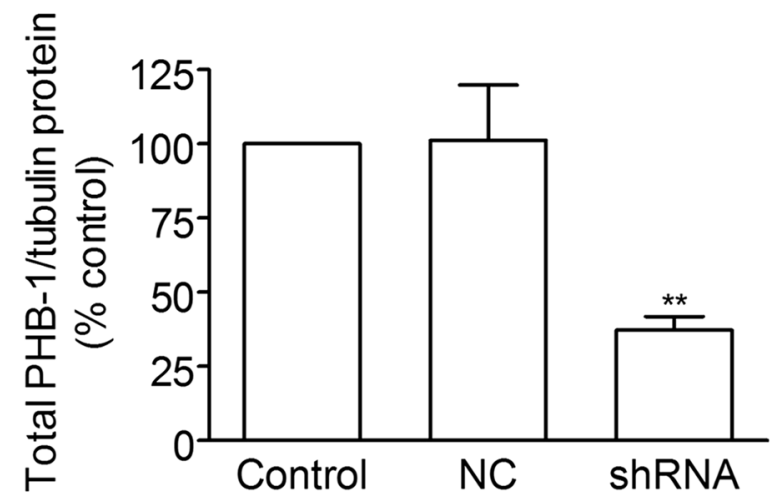

e

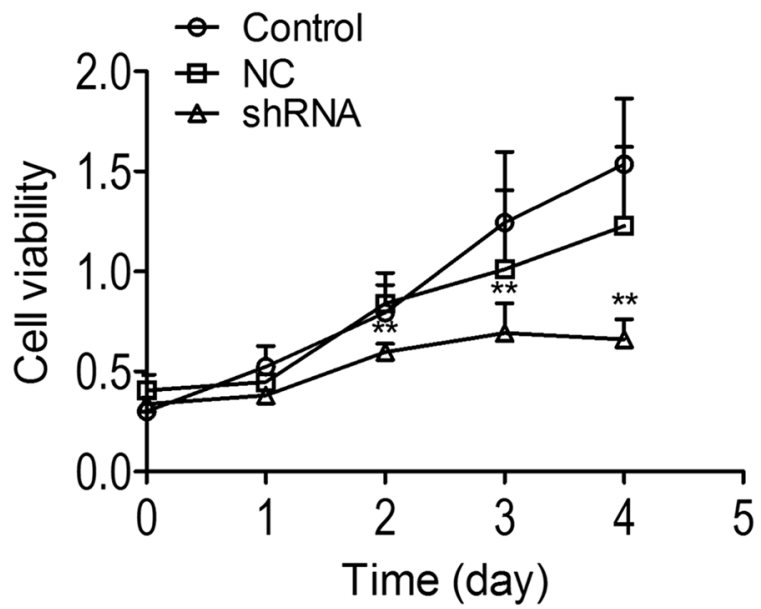

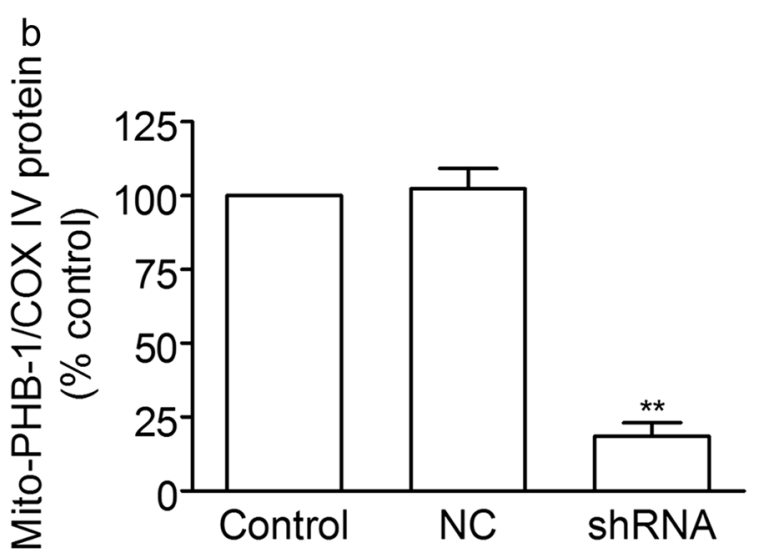

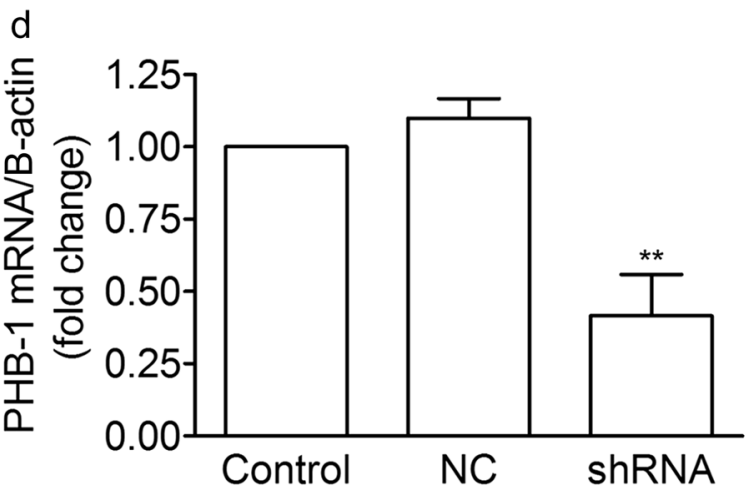

f

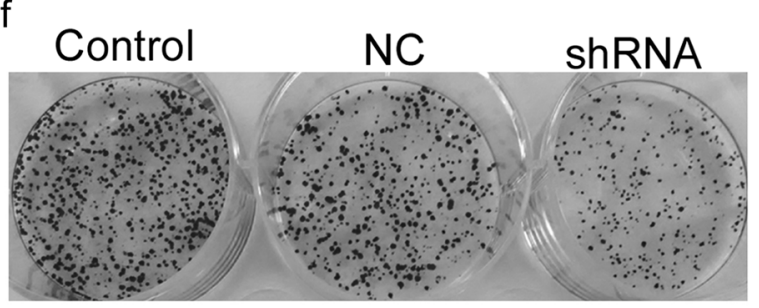

g

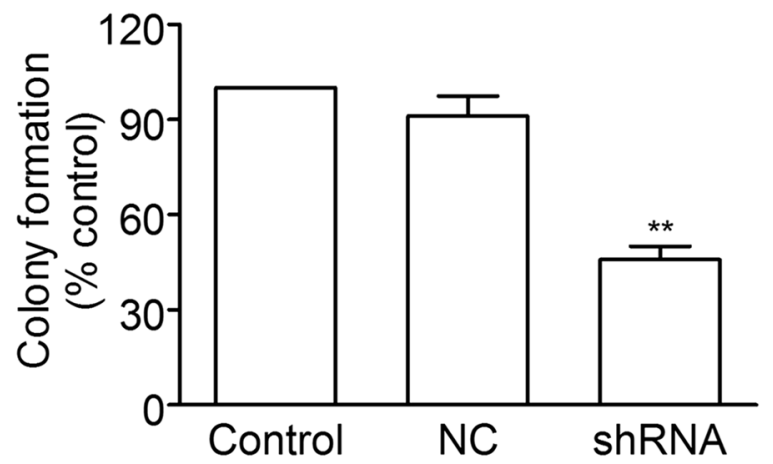

Fig. 7 PHB-1 regulated the growth of human gastric cancer cell line SGC-7901 cells. a Western blot analysis of the expression levels of the mitochondrial and total PHB-1 in the control, scrambled negative control (NC), and shRNA-transfected cultured SGC-7901 cells. Densitometric analysis of bands for relative mitochondrial PHB-1 (b) and total PHB-1 (c) expression levels in the control, NC, and shRNA cells. d Real-time PCR analysis of the PHB-1 mRNA level in the control, NC, and shRNA cells. e Knockdown of PHB-1 (mitochondrial PHB-1) inhibited the growth rate of SGC-7901 cells. $\mathbf{f}$ Representative images of the cloning wells of control, NC, and shRNA cells. $g$ Quantitative image analysis of colonies in control, NC, and shRNA cells. Knockdown of PHB-1 (mitochondrial PHB-1) inhibited the colony formation capacity of SGC-7901 cells. Data are expressed as the mean \pm SD. $n=6 .{ }^{* *} P<0.01$ vs. the control group 
metabolism pathways (including mitochondrial assembly, pyruvate metabolism, (unsaturated) fatty acid $\beta$-oxidation, ketone body catabolism, and oxidative phosphorylation) was identified to be downregulated by carnosine treatment (Table 1).

PHB-1 has been considered to be a multifunctional protein, with effects including anti-proliferation, differentiation, morphogenesis, and transcription. This diverse array of functions of PHB-1 is attributed to the cell type studied and its subcellular localization $[25,26]$. PHB-1 was found to be overexpressed in gastric tumors, and it may be a putative diagnostic and differentiation biomarker for the tissue-based detection of gastric cancer [27]. Recently, Jiang et al. [18] reported that mitochondrial PHB-1 may play an important role in tumorigenesis and suggested that reducing mitochondrial PHB-1 may inhibit tumor progression. In the present study, we found that carnosine treatment caused a marked decrease in the localization of PHB-1 in mitochondria. Interestingly, previous researchers observed that endogenous Akt directly regulates the phosphorylation of PHB-1 Thr258 and mediates the translocation of PHB-1 to the mitochondria in $\mathrm{BC}$ cells [18]. Our study shows that in cultured SGC-7901 and BGC-823 cells, the level of p-Akt was significantly decreased by carnosine treatment, and inhibition of Akt activation by its inhibitor GSK690693 effectively reduced the localization of PHB-1 in mitochondria. Thus, our data also suggest that Akt mediates the mitochondrial localization of PHB-1, and the effects of carnosine may be due at least in part to its ability to suppress the Akt pathway to decrease the mitochondrial localization of PHB-1.

Loss of mitochondrial PHB-1 expression has been shown to lead to impairment of the mitochondrial respiratory chain [28]. In addition, carnosine treatment also affects some mitochondrial respiratory complex proteins (Table 1). Thus, we speculated that carnosine may inhibit the activities of the mitochondrial respiratory chain complexes in SGC-7901 cells by reducing the expression of mitochondrial PHB-1 and some respiratory complex proteins. However, we cannot exclude the possibilities that carnosine may inhibit the activities of complexes I-IV by direct interaction with respiratory chain complexes. In addition, although there are hints that loss of mitochondrial PHB-1 expression could lead to accelerated proteolysis of imported proteins in mitochondria by the m-AAA protease [28], whether the inhibitory effect of carnosine on mitochondrial protein expression is mediated by the reduced expression of mitochondrial PHB- 1 is not known. However, mitochondrial PHB-1 is obviously a positive regulator of cell proliferation in human gastric cancer cells as shPHB transfectants with markedly decreased mitochondrial PHB-1 expression exhibited an inhibited cell growth rate and colony formation capacity compared to controls.

On the other hand, a controversial role of carnosine in fighting mitochondrial dysfunction by relieving oxidative stress in agingrelated pathology has been proposed [29, 30]. Carlo Corona et al. found a very potent effect of carnosine in rescuing mitochondrial dysfunctions in aged 3xTg-AD mice. These authors found that carnosine supplementation not only prevented the reduction in the activity of complex I, II, and IV in the hippocampus and the activity of complex I and IV in the cortex but also enhanced the activity of complexes II and IV in the hippocampus over baseline levels [29]. The underlying mechanisms of the entirely different roles of carnosine in normal and tumor cells remain to be elucidated, and here, we speculated that the different roles may be due to metabolic differences or different signal transduction pathways that could be activated by carnosine treatment in normal and tumor cells.

In conclusion, this study identified multiple mitochondrial proteins that are related to mitochondrial bioenergetics as the targets of carnosine for the first time, and a decrease in the localization of PHB-1 in mitochondria induced by inhibition of Akt phosphorylation may play an important role in the inhibitory action of carnosine in human gastric cancer cells. Our findings demonstrate that carnosine may be a potential agent targeting tumor metabolism to inhibit the growth of human gastric cancer cells both in vitro and in vivo.

\section{ACKNOWLEDGEMENTS}

This work was supported by the National Natural Science Foundation of China (81571289) and Key Discipline of Zhejiang Province in Medical Technology (First Class, Category A).

\section{AUTHOR CONTRIBUTIONS:}

YS and JXL conceived and designed the experiments; JYC, JBY, and YS wrote the manuscript; JYC and JBY performed experiments and analyzed the data; YL, MX, YYH, $J J Z$, and PC performed experiments.

\section{ADDITIONAL INFORMATION}

Competing interests: The authors declare no competing interests.

\section{REFERENCES}

1. Koppenol WH, Bounds PL, Dang CV. Otto Warburg's contributions to current concepts of cancer metabolism. Nat Rev Cancer. 2011;11:325-37.

2. Jia D, Park JH, Jung KH, Levine $H$, Kaipparettu BA. Elucidating the metabolic plasticity of cancer: mitochondrial reprogramming and hybrid metabolic states. Cells. 2018;7:pii: E21.

3. Diers AR, Broniowska KA, Chang CF, Hogg N. Pyruvate fuels mitochondrial respiration and proliferation of breast cancer cells: effect of monocarboxylate transporter inhibition. Biochem J. 2012;444:561-71.

4. Cheng G, Zielonka J, McAllister D, Tsai S, Dwinell MB, Kalyanaraman B. Profiling and targeting of cellular bioenergetics: inhibition of pancreatic cancer cell proliferation. Br J Cancer. 2014;111:85-93.

5. Kwiatkowski S, Kiersztan A, Drozak J. Biosynthesis of carnosine and related dipeptides in vertebrates. Curr Protein Pept Sci. 2018;19:771-89.

6. Boldyrev AA. Problems and perspectives in studying the biological role of carnosine. Biochemistry (Mosc). 2000;65:751-6.

7. Ghodsi R, Kheirouri S. Carnosine and advanced glycation and products: a systematic review. Amino Acids. 2018:50:1177-86.

8. Horii Y, Shen J, Fujisaki Y, Yoshida K, Nagai K. Effects of L-carnosine on splenic sympathetic nerve activity and tumor proliferation. Neurosci Lett. 2012;510:1-5.

9. Shen Y, Yang J, Li J, Shi X, Ouyang L, Tian Y, et al. Carnosine inhibits the proliferation of human gastric cancer SGC-7901 cells through both of the mitochondrial respiration and glycolysis pathways. PLoS ONE. 2014;9:e104632.

10. Shen Y, Tian Y, Yang J, Shi X, Ouyang L, Gao J, et al. Dual effects of carnosine on energy metabolism of cultured cortical astrocytes under normal and ischemic conditions. Regul Pept. 2014;192-193:45-52.

11. Shi L, Wang Y, Tu S, Li X, Sun M, Srivastava $S$, et al. The responses of mitochondrial proteome in rat liver to the consumption of moderate ethanol: the possible roles of aldo-keto reductases. J Proteome Res. 2008;7:3137-45.

12. Spinazzi M, Casarin A, Pertegato V, Salviati L, Angelini C. Assessment of mitochondrial respiratory chain enzymatic activities on tissues and cultured cells. Nat Protoc. 2012;7:1235-46.

13. Manfredi G, Yang L, Gajewski CD, Mattiazzi M. Measurements of ATP in mammalian cells. Methods. 2002;26:317-26.

14. Renner C, Zemitzsch N, Fuchs B, Geiger KD, Hermes M, Hengstler J, et al. Carnosine retards tumor growth in vivo in an NIH3T3-HER2/neu mouse model. Mol Cancer. 2010;9:2.

15. Artal-Sanz M, Tavernarakis N. Prohibitin and mitochondrial biology. Trends Endocrinol Metab. 2009;20:394-401.

16. Peng YT, Chen $\mathrm{P}$, Ouyang RY, Song L. Multifaceted role of prohibitin in cell survival and apoptosis. Apoptosis. 2015;20:1135-49.

17. Sasaki T, Kuniyasu H. Significance of AKT in gastric cancer. Int J Oncol. 2014:45:2187-92.

18. Jiang L, Dong P, Zhang Z, Li C, Li Y, Liao Y, et al. Akt phosphorylates Prohibitin 1 to mediate its mitochondrial localization and promote proliferation of bladder cancer cells. Cell Death Dis. 2015;6:e1660.

19. Li J, Wang X, Hou J, Huang Y, Zhang Y, Xu W. Enhanced anticancer activity of 5-FU in combination with Bestatin: evidence in human tumor-derived cell lines and an H22 tumor-bearing mouse. Drug Discov Ther. 2015;9:45-52.

20. Letzien U, Oppermann H, Meixensberger J, Gaunitz F. The antineoplastic effect of carnosine is accompanied by induction of PDK4 and can be mimicked by L-histidine. Amino Acids. 2014:46:1009-19. 
21. Otani H, Okumura N, Hashida-Okumura A, Nagai K. Identification and characterization of a mouse dipeptidase that hydrolyzes $L$-carnosine. J Biochem. 2005;137:167-75.

22. Hipkiss AR, Gaunitz F. Inhibition of tumour cell growth by carnosine: some possible mechanisms. Amino Acids. 2014;46:327-37.

23. Pathania D, Millard M, Neamati N. Opportunities in discovery and delivery of anticancer drugs targeting mitochondria and cancer cell metabolism. Adv Drug Deliv Rev. 2009;61:1250-75.

24. Renner C, Asperger A, Seyffarth A, Meixensberger J, Gebhardt R, Gaunitz F. Carnosine inhibits ATP production in cells from malignant glioma. Neurol Res. 2010;32:101-5.

25. Chowdhury I, Thompson WE, Thomas K. Prohibitins role in cellular survival through Ras-Raf-MEK-ERK pathway. J Cell Physiol. 2014;229:998-1004.

26. Osman C, Merkwirth C, Langer T. Prohibitins and the functional compartmentalization of mitochondrial membranes. J Cell Sci. 2009;122:3823-30.
27. Kang X, Zhang L, Sun J, Ni Z, Ma Y, Chen X, et al. Prohibitin: a potential biomarker for tissue-based detection of gastric cancer. J Gastroenterol. 2008;43:618-25.

28. Piechota J, Kolodziejczak M, Juszczak I, Sakamoto W, Janska H. Identification and characterization of high molecular weight complexes formed by matrix AAA proteases and prohibitins in mitochondria of Arabidopsis thaliana. J Biol Chem. 2010;285:12512-12512.

29. Corona C, Frazzini V, Silvestri E, Lattanzio R, La Sorda R, Piantelli M, et al. Effects of dietary supplementation of carnosine on mitochondrial dysfunction, amyloid pathology, and cognitive deficits in 3xTg-AD mice. PLoS ONE. 2011;6: e17971.

30. Hipkiss AR. Aging, proteotoxicity, mitochondria, glycation, NAD + and carnosine: possible inter-relationships and resolution of the oxygen paradox. Front Aging Neurosci. 2010;2:10. 